\section{Possible Chromatographic Effect of Liquid-Crystalline Permeation}

\section{W. Helfrich}

Institut für Theoretische Physik der Kondensierten Materie Freie Universität Berlin

Berlin, Arnimallee 3, West Germany

(Z. Naturforsch. 28 a, $1967-1968$ [1973] ; received October 23, 1973)

Permeation, i.e. material flow through cholesteric and smectic layers, may vary in speed for the components of a mixture. We derive qualitative formulas for the permeation rate of solute molecules in cholesterics as a function of their size. The possibility of a new kind of chromatography based on permeation in cholesterics or smectics is discussed.

Because of their layered structures cholesteric and smectic liquid crystals are capable of displaying permeation or pluglike flow, i. e. a material transport through the layers ${ }^{1}$. The migrating molecules rotate around the helixal axis in cholesterics and jump between monomolecular layers in smectics. If the liquid crystal comprises more than one kind of molecules the various species may permeate at different velocities. This is reminiscent of chromatography. In the present note we wish to explore the possibility of using permeation for chromatographic purposes. The arrangement to be considered is a capillary tube filled with a cholesteric or smectic liquid crystal, the layers being normal to its axis. A pressure gradient along the capillary provides the driving force of permeation.

With cholesterics we have for the permeation velocity $v$ of a one-component material ${ }^{1}$

$$
v=-\frac{1}{\gamma}\left(\frac{2 \pi}{P}\right)^{2} p^{\prime}
$$

where $\gamma$ is the rotational viscosity, $P$ the cholesteric pitch, and $p^{\prime}$ the pressure gradient. The formula was derived on the assumption that the pitch is much smaller than the radius of the capillary (or an equivalent measure in the case of noncircular cross sections).

If a solute is added in small enough concentrations, Eq. (1) will still be valid for the solvent. To obtain a formula for the velocity of the solute we imagine a single guest particle which can be treated as macroscopic and moves initially with the same translational speed as the host material. The alignment of the guest may be either locked to that of the host or free. In either event the cholesteric orientation pattern is assumed to be essentially undisturbed by the presence of the guest. The rotation of the

Reprint requests to Prof. Dr. W. Helfrich, Institut für Theoret. Physik, Freie Universität Berlin, D-1000 Berlin 33, Arnimallee 3. host molecules exerts the torque

$$
t_{1}=-\gamma V(2 \pi / P) v .
$$

on the guest where $V$ is the volume of the guest particle.

In the case of locked alignment an elastic torque sustained by the liquid-crystalline orientation pattern balances the frictional torque $t_{1}$. The associated elastic force, $-t_{1}(2 \pi / P)$, offsets the pressure force $-V p^{\prime}$ in precisely the same way as in the host material. However, the rotation of the moving guest particle induces a shear flow around it. According to Stokes ${ }^{3}$ the associated torque $t_{2}$ felt by the particle would be

$$
t_{2}=-8 \pi \eta R^{3} \omega
$$

if one would be dealing with a sphere of radius $R$ in an isotropic liquid. Here $\eta$ is the viscosity of the medium and $\omega$ the angular velocity of the rotation. The frictional torque $t_{2}$ must be compensated by an additional elastic torque which, in turn, is connected with the elastic force $-t_{2}(2 \pi / P)$. This force results in a slowing of the guest motion. In our picture of a sphere in an isotropic fluid the velocity $v_{\mathrm{g}}$ of the guest obeys the relation

$$
v_{\mathrm{g}}-v=-\frac{4}{3} R^{2}\left(\frac{2 \pi}{P}\right)^{2} v_{\mathrm{g}}
$$

where use is made of another, well-known formula by Stokes (force $=6 \pi \eta R$. velocity).

Our description is incomplete in that the shear flow around a rotating body cannot reach far into the liquid-crystalline medium because it produces torque and force densities. As a consequence, the force $-t_{2}(2 \pi / P)$ exerted on the body must be associated with an equal but opposite sum of forces acting on the surroundings. Simple reasoning indicates that the bulk of these forces should occur in the range $R<r<2 R$, where $r$ is the distance from the center of the sphere. While the counterforces will reduce $v-v_{\mathrm{g}}$ by a certain fraction, they cannot make it vanish so that (4) remains approximately valid.

If the alignment of the guest particle is free, it is advantageous to start from the notion of a guest particle which moves at the translational speed of the host, but does not rotate. Neither the torque $t_{1}$ nor the pressure force $-V p^{\prime}$ acting on the particle can now be offset elastically. The unbalanced pressure force causes the particle to move faster than the host. For a sphere in an isotropic fluid the resulting velocity $v_{\mathrm{g}}$ is given by

$$
v_{\mathrm{g}}-v=\frac{V p^{\prime}}{6 \pi \eta R}=\frac{2}{9} R^{2}\left(\frac{2 \pi}{P}\right)^{2} v .
$$


For the second form we have eliminated $p^{\prime}$ by use of Eq. (1), putting $\gamma=\eta$. The torque makes the sphere turn around the helical axis, the rotations of host and guest being opposite to each other. The shear flow around the rotating particle generates a force equal but opposite to $-V p^{\prime}$ in the surroundings, thus diminishing the relative velocity $v_{\mathrm{g}}-v$.

The simple relationships (4) and (5) seem useful for a qualitative discussion. They are, of course, not exact. Regarding the guest particle as macroscopic is a gross simplification if solvent and solute molecules are comparable in size. Furthermore, the macroscopic description takes no account of nonspherical particle shapes and of the viscosity anisotropy of liquid crystals. Obviously, a correction of these and other defects would be very difficult. It should also be mentioned that in deriving (4) and (5) we have tacitly assumed $R \ll P$. Considering a macroscopic particle of locked alignment, one readily convinces oneself that regardless of size the velocity $v_{g}$ cannot be negative, i. e. opposite to the pressure force. Particles as large as the pitch or larger are very unlikely to be free in their alignment. Therefore, we do not go into the complexities of permeation of large $(R \gtrsim P)$ unlocked particles.

Our results suffice to conclude that permeation in cholesterics permits in principle a new kind of chromatography. The separation of different species of molecules is probably most effective if they are much larger than the host. At least with locked alignment they should also be smaller than the cholesteric pitch. If both species are either very large or very small, their rates of permeation will be almost equal being either like that of the host or practically zero.

Very little can be predicted about permeation in smectic liquid crystals. We may expect the molecules of a one-component material to jump with a certain frequency $v$ between adjacent monomolecular layers. Since there will be an energy barrier we may write

$$
v=v \exp \left\{-E / k_{\mathrm{B}} T\right\} .
$$

However, the activation energy $E$ should depend on the degree of liquid-crystalline order, thus being itself a function of temperature. Let us assume here that the barrier is at its peak if the center of mass of the jumping molecule is just midway between layers.

1 W. Helfrich, Phys. Rev. Letters 23, 372 [1969]. In another article [W. Helfrich, in: Liquid Crystals and Ordered Fluids (Plenum Press, New York 1970) p. 405] a circular flow in round capillaries on top of permeation was erroneously calculated for cholesterics. Actually, there can be no circular flow as permeation does not produce "circular" forces. (See, e. g., Ref. ${ }^{2}$.) We remark
Then we have for the permeation velocity under the action of a pressure gradient ${ }^{1}$

$$
v=-v a^{2} V p^{\prime} / k_{\mathrm{B}} T
$$

where $\mathrm{a}$ is the layer spacing. This is in close analogy to a well-known formula for electric conduction by carrier hopping. The jumping frequencies may be anticipated to vary over a very wide range. To our knowledge, no data have as yet been reported.

A guest molecule can move faster or slower than the host, depending on its relative jumping frequency and volume. (We do not attempt here an exact definition of the effective volume $V$ in (7). We also disregard multiple jumps involving more than one molecule or carrying farther than to the next layer.) As a method to separate different species of molecules permeation in smectics is likely to be most effective if the dissolved molecules are small. The bigger the molecule, the more prohibitive the energy barrier for jumping can become.

In summary, it seems that cholesteric chromatography may be a means to separate molecules of very large molecular weight for which conventional chromatography does not work. Smectics could be useful as highly selective and adaptable sieves for smaller molecules.

Although liquid-crystalline chromatography appears attractive in theory, putting it into practice will not be easy. In the first place one has to establish pluglike flow in capillaries with firm anchoring of the cholesteric or smectic layers on the capillary wall. There are some encouraging data in the literature ${ }^{4}$ which indicate capillary flow by permeation up to fairly high pressure gradients (ca. $1 \mathrm{~atm} \mathrm{~cm}^{-1}$ ) in low pitch cholesterics $(P \approx 3000 \AA)$ and in smectics. Another practical problem is the filling and emptying of the capillary before and after the chromatographic process. Essential from a general point of view is the requirement that the pressure force be large enough to make transport faster than diffusion. It is expressed mathematically by the condition

$$
-V p^{\prime} L \gg k_{\mathrm{B}} T
$$

where $L$ is the length of the capillary. Accordingly, very large pressure drops $-p^{\prime} L$ which may not be practicable are needed for small molecules.

that "circular" forces do occur directly at the capillary wall in a layer of Poiseuille shear.

2 T. C. Lubensky, Phys. Rev. A 6, 452 [1972].

3 See, e. g., H. Morawetz, Macromolecules in Solution, Interscience Publishers, New York 1965.

4 R. S. Porter, E. M. Barrall, II, and J. F. Johnson, J. Chem. Phys. 45, 1452 [1966]. 